Schrödinger equation for the one-particle density matrix of thermal systems: an alternative formulation of Bose-Einstein condensation

This article has been downloaded from IOPscience. Please scroll down to see the full text article. 2007 J. Phys. A: Math. Theor. 40 F243

(http://iopscience.iop.org/1751-8121/40/10/F03)

View the table of contents for this issue, or go to the journal homepage for more

Download details:

IP Address: 128.206.162.204

The article was downloaded on 04/06/2010 at 17:57

Please note that terms and conditions apply. 
FAST TRACK COMMUNICATION

\title{
Schrödinger equation for the one-particle density matrix of thermal systems: an alternative formulation of Bose-Einstein condensation
}

\author{
Subodha Mishra and Peter Pfeifer \\ Department of Physics \& Astronomy, University of Missouri, Columbia, MO 65211, USA
}

Received 17 November 2006

Published 21 February 2007

Online at stacks.iop.org/JPhysA/40/F243

\begin{abstract}
We formulate a linear Schrödinger equation with a temperature-dependent potential for the one-particle density matrix and obtain the condensation temperature of the Bose-Einstein condensate from a bound-state condition for the Schrödinger equation both with and without the confining trap. The results are in very good agreement with those of the full statistical physics treatment. This is an alternative to the Bose-Einstein condensation in the standard ideal Bose-gas treatment.
\end{abstract}

PACS numbers: $03.75 . \mathrm{Nt}$, 03.75.Hh, 05.30.-d

\section{Introduction}

Based on the Bose-Einstein statistics [1], Einstein predicted [2] that for a gas of noninteracting, massive bosons, below a critical temperature a nonzero fraction of the total number of particles would occupy the lowest energy single-particle state forming a condensate. This phenomenon of Bose-Einstein condensation (BEC) of dilute gases has been extensively studied theoretically [3] and only recently it has been experimentally [4-6] realized for dilute atomic gases of ${ }^{87} \mathrm{Rb},{ }^{7} \mathrm{Li},{ }^{23} \mathrm{Na}$. It is known that the Gross-Pitaevskii equation (GPE) [7], a nonlinear Schrödinger equation for the macroscopic wavefunction of the BEC provides a detailed description of its ground state spectrum. Though the GPE has been solved variationally $[8,9]$, in this paper, we give a new theoretical treatment of the BEC by incorporating the Bose statistics of the system with a temperature-dependent potential and solving a linear temperature-dependent Hamiltonian variationally. By incorporating a nonlinear term like $\sim|\psi|^{2}$ in our Hamiltonian one can construct a finite temperature extension of the GrossPitaevskii equation for an interacting bose gas. The present approach can be extended in many ways including the study of temperature dependence of the energy of a vortex in a Bose gas and effect of temperature on the interference of two macroscopic Bose condensates. The 
free-particle many-body Hamiltonian [10] with the quantum correction due to the Bose/Fermi statistics of the system is given as a function of temperature as

$$
H=\sum_{i} \frac{p_{i}^{2}}{2 m}+\sum_{i, j, i \neq j} \tilde{v}\left(\left|\vec{r}_{i}-\vec{r}_{j}\right|\right),
$$

where $\vec{p}_{i}$ and $\vec{r}_{i}$ are the momentum and position of the $i$ th particle, the potential is given by

$$
\tilde{v}(r)=-k_{B} T \ln \left[1 \pm \exp \left(-2 \pi \frac{r^{2}}{\lambda^{2}}\right)\right]
$$

and $\lambda$ is the thermal wave length, $\lambda=\sqrt{2 \pi \hbar^{2} / m k_{B} T}$. The plus sign is for bosons, and the minus sign is for fermions. The Hamiltonian (1) represents the first quantum correction to the classical ideal gas (Boltzman statistic) and describes the system in the range where the interparticle separation is much greater than the corresponding thermal wavelength, $\left|\vec{r}_{i}-\vec{r}_{j}\right| \gg \lambda$. The statistical potential $\tilde{v}(r)$ is attractive for bosons and repulsive for fermions. The one-particle Schrödinger equation is given as

$$
\frac{-\hbar^{2}}{2 \mu} \nabla^{2} \psi+\tilde{v} \psi=E \psi,
$$

where $\mu=m / 2$ is the reduced mass of the two-particle system when the Hamiltonian equation (1) is written for $i=2$. The exact thermal one-particle density matrix [11] for Bose systems, in position representation is given as

$$
\begin{aligned}
\rho_{1}\left(\left|\vec{r}-\vec{r}^{\prime}\right|, T\right) & =F\left(\left|\vec{r}-\vec{r}^{\prime}\right|, T\right) \\
& =\frac{1}{\lambda^{3}} \sum_{j=1}^{\infty} j^{-\frac{3}{2}} \exp \left(-\alpha j-\pi r^{2} / j \lambda^{2}\right),
\end{aligned}
$$

where $\alpha$ has the value $0<\alpha \leqslant 1$ and $r=\left|\vec{r}-\vec{r}^{\prime}\right|$. Our claim is that the leading term in the oneparticle density matrix of a bose system in the position representation can be approximated as $\rho_{1}^{L}(r, T) \approx|\psi(r, T)|^{2}$ where $L$ denotes the leading term and $\psi$ is the grand state of equation (3). We replace the one-particle Hamiltonian $\frac{-\hbar^{2}}{2 \mu} \nabla^{2}+\tilde{v}$ by

$$
\begin{aligned}
& H_{\text {eff }}:=\frac{-\hbar^{2}}{2 \mu} \nabla^{2}+\epsilon \tilde{\tilde{v}} \\
& \tilde{\tilde{v}}:=-k_{B} T \mathrm{e}^{-\frac{2 \pi r^{2}}{\lambda^{2}}}
\end{aligned}
$$

where we have approximated $\tilde{v}(r) \approx \tilde{v}(r)$ for large $T$, that is when the interparticle separation is much greater than the corresponding thermal wavelength, $r \gg \lambda$. We have multiplied the potential with $\epsilon$ to take into account the higher order correlation effect, and it will be determined dynamically such that the eigenvalue of the Hamiltonian is $\leqslant 0$. We derive the dependence of critical condensation density on temperature of the system with and without a confining trap. We state an alternate derivation of the quantum potential and analyse our wavefunction in the limiting case in the appendix.

\section{Schrödinger's equation in 3D}

The time-independent 3D Schrödinger equation in spherical polar coordinates for the reduced mass $\mu=m / 2$ is given as

$$
\begin{gathered}
\frac{-\hbar^{2}}{2 \mu}\left[\frac{1}{r^{2}} \frac{\partial}{\partial r} r^{2} \frac{\partial}{\partial r}+\frac{1}{r^{2} \sin \theta} \frac{\partial}{\partial \theta}\left(\sin \theta \frac{\partial}{\partial \theta}\right)+\frac{1}{r^{2} \sin ^{2} \theta} \frac{\partial^{2}}{\partial \phi^{2}}+\tilde{\tilde{v}}(r, T)\right] \psi(r, \theta, \phi) \\
=E \psi(r, \theta, \phi),
\end{gathered}
$$


where $E$ is the energy eigenvalue corresponding to the wavefunction. We write the total wavefunction $\psi(r, \theta, \phi)$ as a product of the radial and the angular parts:

$$
\psi(r, \theta, \phi)=R_{n, l}(r) Y_{l, m}(\theta, \phi) .
$$

Substituting the above $\psi(r, \theta, \phi)$ into the 3D Schrödinger equation, we get the equation for $R_{n, l}(r)$ as

$$
\frac{1}{r^{2}} \frac{\partial}{\partial r}\left(r^{2} \frac{\partial R}{\partial r}\right)+\frac{2 \mu}{\hbar^{2}}\left[E-\tilde{\tilde{v}}(r, T)-\frac{l(l+1) \hbar^{2}}{2 \mu r^{2}}\right] R(r)=0
$$

Using $R(r)=\frac{\chi(r)}{r}$, we get the standard 1D radial Schrödinger equation as

$$
-\frac{\hbar^{2}}{2 \mu} \frac{\partial^{2} \chi}{\partial r^{2}}+\left[\tilde{\tilde{v}}(r, T)+\frac{l(l+1) \hbar^{2}}{2 \mu r^{2}}\right] \chi=E \chi .
$$

\section{BEC without harmonic trap}

\subsection{Variational calculation}

First we study the simple case of the BEC without any confining trap. For $l=0$, the 1D Hamiltonian is given from equation (10) as

$$
H_{\text {eff }}=\frac{-\hbar^{2}}{2 \mu} \frac{\partial^{2}}{\partial r^{2}}-\epsilon k_{B} T \exp \left(-\frac{2 \pi r^{2}}{\lambda^{2}}\right) .
$$

We choose the trial wavefunction $\chi(r)$ as

$$
\chi(r, T)=A r \mathrm{e}^{-\delta r^{2}},
$$

where $\delta$ is a variational parameter which will depend on temperature $T$, and $A$ is the normalization constant, given as

$$
A=\left(\frac{2^{7} \delta^{3}}{\pi}\right)^{\frac{1}{4}}
$$

Now we calculate the expectation value of the Hamiltonian $H_{\text {eff }}$ given by equation (11), which gives us the energy

$$
E=\left\langle\chi(r, T)\left|H_{\mathrm{eff}}\right| \chi(r, T)\right\rangle .
$$

We get the energy $E$ as

$$
E=k_{B} T\left[\frac{3 \delta}{2 \pi \alpha}-\epsilon\left(\frac{1}{1+\pi \alpha \delta^{-1}}\right)^{(3 / 2)}\right],
$$

where $\alpha=\frac{m k_{B} T}{2 \pi \hbar^{2}}$ and $\delta / \alpha$ is dimensionless. Now we do the minimization of the energy given by equation (15) with respect to $\delta$, which is $\frac{\partial E(\delta, \epsilon)}{\partial \delta}=0$. We get

$$
\frac{\delta}{\pi \alpha}-\epsilon^{2 / 5}\left(\frac{\delta}{\pi \alpha}\right)^{1 / 5}+1=0 .
$$

Since the bound state starts to appear when $E=0$ and the above minimum condition is 


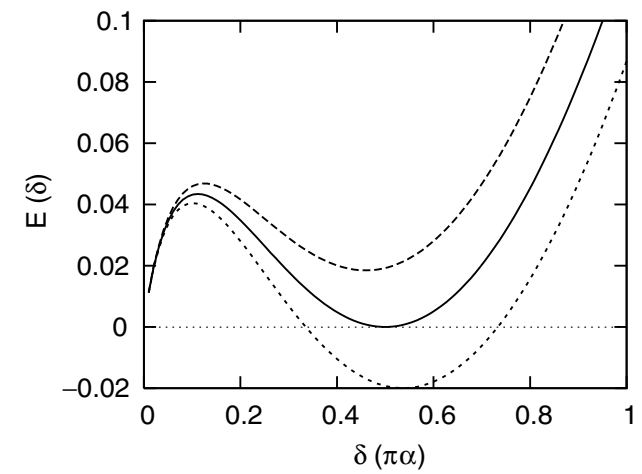

Figure 1. Variation of ground state energy of the bose gas with the parameter $\delta$ for different values of $\epsilon$. For $\epsilon=3.897$, the curve with the solid line shows that the ground state just begins to form. The long dashed line is for $\epsilon=3.797$ and the small dashed line is for $\epsilon=3.997$.

satisfied, we solve the two simultaneous equations for the two unknowns $\delta$ and $\epsilon$ as

$$
\begin{aligned}
& E(\epsilon, \delta)=0 \\
& \frac{\partial E(\epsilon, \delta)}{\partial \delta}=0
\end{aligned}
$$

and we get $\epsilon=\epsilon_{c}=3.897$ and $\delta=\delta_{c}=0.5 \pi \alpha$. In the next section we will see that this set of values of $\epsilon, \delta$ gives the right relation between density and temperature. The plot of $E(\delta)$ as a function of $\delta$ for different values of $\epsilon$ is given in figure 1. This shows for $\epsilon=\epsilon_{c}=3.897, \delta=\delta_{c}=0.5 \pi \alpha$ the bound state just begins to form which is the onset of BEC (shown as solidline).

\subsection{The density-temperature relation}

We now calculate the density of an uniform bose gas in a volume $v$ at temperature $T$ at which the condensation occurs. We have effectively one particle in volume $v$. We use the standard definition of volume as $v=\left\langle r^{3}\right\rangle$ i.e.,

$$
v=\left(\frac{\int r^{3}|\psi(r)|^{2} \mathrm{~d}^{3} r}{\int|\psi(r)|^{2} \mathrm{~d}^{3} r}\right)=\left(\frac{\int r^{3}|\chi(r)|^{2} \mathrm{~d} r}{\int|\chi(r)|^{2} \mathrm{~d} r}\right)=\left(\frac{2}{\pi \delta^{3}}\right)^{\frac{1}{2}}
$$

and

$$
n_{c}=\frac{1}{v}=2.467\left(\frac{m k_{B} T_{c}}{2 \pi \hbar^{2}}\right)^{\frac{3}{2}} .
$$

We see from equation (20) that we obtain the correct relation between condensate density $n_{c}$ of the bose gas and the temperature $T_{c}$ at which the condensation occurs from an effective one-particle picture. The exact relation has the constant [10] $g_{3 / 2}(1)=\zeta(3 / 2)=2.612$, where $\zeta(x)$ is the Riemann zeta function of $x$. The density-temperature relation is the most important result of our paper. Since our treatment is variational the coefficient is a little off from the exact value. This shows that our formalism reproduces the relation which is known for an ideal bose gas [10]. 


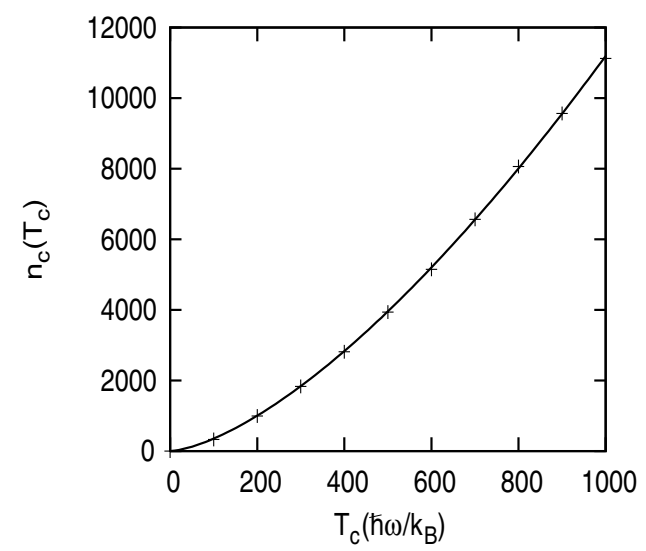

Figure 2. Variation of condensation density with temperature. The +'s are the numerically calculated data and the solid line is a fit function given as $0.354 T^{3 / 2}$.

\section{BEC in the harmonic trap}

Now we study the BEC in an isotropic harmonic trap. The Hamiltonian for the quantum bose gas in the trap is given as

$$
H=\frac{-\hbar^{2}}{2 \mu} \frac{\partial^{2}}{\partial r^{2}}-\epsilon k_{B} T \exp \left(-\frac{2 \pi r^{2}}{\lambda^{2}}\right)+\frac{1}{2} \mu \omega^{2} r^{2}
$$

We have added the extra confining potential with frequency $\omega$ to our original Hamiltonian (equation (11)) and we want to study its effect on the BEC. We use our trial wavefunction given by equation (12) with the normalization constant $A$ given by equation (13). We calculate the energy $E$ and minimize it to get the two equations for the two parameters $\epsilon$ and $\delta$. The two equations are given as

$$
\begin{aligned}
& E=k_{B} T\left[\frac{3 \delta}{2 \pi \alpha}-\frac{\epsilon}{\left(1+\pi \alpha \delta^{-1}\right)^{\frac{3}{2}}}+\frac{3 \sqrt{2}}{8}\left(\frac{\hbar \omega}{k_{B} T}\right)^{2}\left(\frac{\pi \alpha}{\delta}\right)\right] \\
& \frac{\partial E}{\partial \delta}=k_{B} T\left[\frac{3}{2 \pi \alpha}-\frac{\epsilon 3(\pi \alpha / \delta)^{2}}{2 \pi \alpha\left(1+\pi \alpha \delta^{-1}\right)^{\frac{5}{2}}}-\frac{3 \sqrt{2}}{8 \pi \alpha}\left(\frac{\hbar \omega}{k_{B} T}\right)^{2}\left(\frac{\pi \alpha}{\delta}\right)^{2}\right] .
\end{aligned}
$$

As before, we get $E=0$ and $\frac{\partial E}{\partial \delta}=0$ to solve simultaneously for the two unknowns $\epsilon$ and $\delta$ at different values of temperature $T$ while keeping the value of frequency $\omega$ constant. Since now we cannot solve for $\delta$ in a closed analytic form as a function of $T$, we do the calculation numerically. For each given value of temperature $T$, we solve for $\epsilon$ and $\delta$. Then using equation (19), we calculate the volume $v$ and condensation density $n_{c}$ for each value of $\delta$ for a set of values of $T$. We show in figure 2 the numerical data obtained for $n_{c}$ versus $T_{c}$ and the fitting of the data with a function $0.354 T^{3 / 2}$, where we have taken $\hbar \omega / k_{B}=1$. The fitting is amazingly good. From the ideal bose-gas treatment the value of the coefficient is 0.167 instead of 0.354 . Since our treatment is variational, the coefficient is overestimated by a factor of 2. Otherwise our formulation correctly reproduces the density-temperature relation for the bose gas confined in a harmonic trap. Since we know that size [12] of the thermal cloud is given by $r \sim\left(\frac{k_{B} T}{m \omega^{2}}\right)^{\frac{1}{2}}$, using this in equation (20) i.e, $N \sim r^{3} n_{c}$, we get the number of particles 
$N$ vary as $T_{c}^{3}$. This is the relation one gets if one uses other methods [12] for the calculation. This shows that the single particle representation of the BEC is very good.

\section{Relation of our trial wavefunction to the exact one-particle density matrix}

For an ideal Bose-Einstein gas it has been shown [11] that the one-particle density matrix is given by equation (4). Since $0<\alpha \leqslant 1$, we can see that the leading term in the above density matrix with $j=1$ and $\alpha=1$ (onset of condensation) has the dependence on $r$ and $T$ as

$$
\rho_{1}^{L}(r) \sim \frac{1}{\lambda^{3}} \mathrm{e}^{-\frac{\pi r^{2}}{\lambda^{2}}}
$$

We can also calculate the one-particle density matrix from our trial wavefunction equation (12), as

$$
\rho_{1}^{\text {our }}(r)=|\psi(r)|^{2}=\left|\frac{\chi(r)}{r} Y_{0,0}\right|^{2}=\frac{1}{\lambda^{3}} \mathrm{e}^{-\frac{\pi r^{2}}{\lambda^{2}}} .
$$

Comparing equations (24) and (25) we see that the functional dependence on $r$ and $T$ of both the density matrices are same. This justifies our description in terms of a temperature-dependent pure state of BEC.

\section{Conclusion}

In conclusion, we have shown that it is possible to get Bose-Einstein condensation with and without the harmonic trap by considering a Hamiltonian with temperature-dependent potential and a temperature-dependent pure state. We obtain the correct relation between condensate density and temperature at the critical point. This is an alternative way to the Bose-Einstein condensation in standard ideal Bose-gas treatment. By incorporating a nonlinear term like $\sim|\psi|^{2}$ in the linear Schrödinger equation one can construct a finite temperature extension of the Gross-Pitaevskii equation [7]. The present approach can be applied to study the temperature dependence of the energy of a vortex in a Bose gas and effect of temperature on the interference of two macroscopic Bose condensates.

\section{Appendix A. Derivation of the interaction potential from the distribution function}

For a pair of non-interacting particles in a volume $v$ the canonical partition function is given as

$$
Z(v, T) \approx \frac{1}{2}\left(\frac{v}{\lambda^{3}}\right)^{2}
$$

where $\lambda$ is the thermal wave length defined before. It can be shown [13] that the probability distribution for the separation $r$ is given as

$$
P_{12}=\frac{1}{v^{2}}\left[1 \pm \exp \left(-2 \pi r^{2} / \lambda^{2}\right)\right]
$$

Now we take the logarithm of the above equation and get

$$
\ln P_{12}=\ln \left[1 \pm \exp \left(-2 \pi r^{2} / \lambda^{2}\right)\right]
$$

where $+(-)$ sign is for boson(fermion). In writing the above equation we have dropped the constant term independent of $r$. We multiply by $-k_{B}$ to find a quantity which has the meaning of an entropy which is given as

$$
\mathcal{S}=-k_{B} \ln \left[1 \pm \exp \left(-2 \pi r^{2} / \lambda^{2}\right)\right]
$$


Now if we multiply the entropy $\mathcal{S}$ with temperature $T$ we have an energy, which here appears as the interaction potential energy.

$$
\mathcal{V}=\mathcal{S} T=-k_{B} T \ln \left[1 \pm \exp \left(-2 \pi r^{2} / \lambda^{2}\right)\right] .
$$

This is exactly the interaction potential [10] used in our theory.

\section{Appendix B. Behaviour of the wavefunction when $T \rightarrow \infty$ and $r \rightarrow 0$}

The interaction potential equation (6) in the limit of high $T$ behaves [14] as

$$
\lim _{T \rightarrow \infty} \tilde{\tilde{v}}(r, T)=-T^{\frac{1}{4}} \delta(r) .
$$

And our trial wavefunction equation (12) behaves [14] in the limit of high $T$ as

$$
\lim _{T \rightarrow \infty} \chi(r, T)=c T^{\frac{1}{4}} r \delta(r),
$$

where $c$ is a function of $\hbar$ and $m$ and independent of $r$ and $T$. If we use the high $T$ limit of the interaction potential equation (B.1) in the 1D radial Schrödinger equation (10), the above high $T$ limit of $\chi(r, T)$ (equation (B.2)) does satisfy the equation with an eigenvalue that diverges. This is exactly the behaviour of the variational energy value (equation (15)) which also diverges in the high limit of $T$.

\section{References}

[1] Bose S N 1924 Z. Phys. 26178

[2] Einstein A 1924 Sitzber. Kgl. Preuss. Akad. Wiss 261 Einstein A 1925 Sitzber. Kgl. Preuss. Akad. Wiss 3

[3] Legget A J 2001 Rev. Mod. Phys. 73307

[4] Anderson M H, Ensher J R, Matthews M R, Wieman C E and Cornell E A 1995 Science 269198

[5] Bradley C C, Sackett C A, Tollett J J and Hulet R G 1995 Phys. Rev. Lett. 751687

[6] Davis K B, Mewes M O, Andrew M R, van Druten N J, Durfee D S, Kurn D M and Ketterle W 1995 Phys. Rev. Lett. 753969

[7] Gross E P 1961 Nuovo Cimento 20454

Gross E P 1963 J. Math. Phys. 4195

Pitaevskii L P 1961 Zh. Eksp. Teor. Fiz. 40646

Pitaevskii L P 1961 Sov. Phys._JETP 13451

[8] Shi H and Zheng W M 1997 Phys. Rev. A 552930

[9] Ueda M and Legget A J 1998 Phys. Rev. Lett. 801576

[10] Uhlenbeck G E and Gropper L 1932 Phys. Rev. 4179

Huang K 1987 Statistical Mechanics 2nd edn (New York: Wiley)

[11] Ziff R M, Uhlenbeck G E and Kac M 1977 Phys. Rep. 32169

[12] Pethick C J and Smith H 2002 Bose-Einstein Condensation in Dilute Gases (Cambridge: Cambridge University Press)

[13] Pathria R K 1996 Statistical Mechanics 2nd edn (New York: Butterworth-Heineman)

[14] Arfken G 1985 Mathematical Methods in Physics (San Diego: Academic) 